\title{
ON SEMIREGULARIZATION \\ OF SOME ABSTRACT DENSITY TOPOLOGIES INVOLVING SETS HAVING THE BAIRE PROPERTY
}

\author{
Jacek Hejduk — Renata Wiertelak — Wojciech Wojdowski
}

\begin{abstract}
Some kind of abstract density topology in a topological Baire space is considered. The semiregularization of this type of topology on the real line in many cases is the coarsest topology for which real functions continuous with respect to the abstract density topology are continuous.
\end{abstract}

Let $\langle X, \tau\rangle$ be a topological Baire space and $\mathbb{K}(\tau), \mathcal{B} a(\tau)$ denote the family of all meager sets and the family of all sets having the Baire property in $\langle X, \tau\rangle$, respectively. By $\mathcal{B}(\tau)$ we shall denote the family of Borel sets in $\langle X, \tau\rangle$. If $A \subset X$, then $\bar{A}^{\tau}$ is $\tau$-closure of $A$ and $\operatorname{int}_{\tau}(A)$ is $\tau$-interior of $A$.

Let $\tau_{0}$ stand for the natural topology on $\mathbb{R}$. The family of meager sets, sets having the Baire property and Borel sets in $\left\langle\mathbb{R}, \tau_{0}\right\rangle$ will be denoted by $\mathbb{K}, \mathcal{B} a$ and $\mathcal{B}$, respectively. Then $\bar{A}$ is $\tau_{0}$-closure and $\operatorname{int}(A)$ is $\tau_{0}$-interior of the set $A \subset \mathbb{R}$.

Let $\lambda$ be the Lebesgue measure on $\mathbb{R}$ and $\mathcal{L}$ the family of Lebesgue measurable sets. We write $|J|$ for the length of an interval $J$. We denote by $\Phi_{d}$ the density operator on $\mathcal{L}$ and by $\mathcal{T}_{d}$ the density topology on $\mathbb{R}$.

If $\mathcal{C}, \mathcal{D}$ are families of subsets of $X$, then

$$
\mathcal{C} \ominus \mathcal{D}=\{A \subset X: A=C \backslash D, C \in \mathcal{C}, D \in \mathcal{D}\} .
$$

As usual, $A \triangle B=(A \backslash B) \cup(B \backslash A)$ for any sets $A, B \subset X$.

By $\mathcal{C}(\langle X, \tau\rangle)$ we shall denote the family of all continuous transformations from $\langle X, \tau\rangle$ to $\left\langle\mathbb{R}, \tau_{0}\right\rangle$. At this moment, it is worth mentioning the following theorem.

TheOREm 1 (cf. 9]). Let $\langle X, \tau\rangle$ be an arbitrary topological Baire space and $\mathcal{J}$ be a proper $\sigma$-ideal of subsets of $X$ free from nonempty $\tau$-open sets and such that the family $\tau \ominus \mathcal{J}$ forms a topology. Then,

$$
\mathcal{C}(\langle X, \tau\rangle)=\mathcal{C}(\langle X, \tau \ominus \mathcal{J}\rangle)
$$

(C) 2016 Mathematical Institute, Slovak Academy of Sciences. 2010 Mathematics Subject Classification: 54A05, 28 A05.

Keywords: lower density operator, abstract density topology, Baire space. 


\section{JACEK HEJDUK — RENATA WIERTELAK — WOJCIECH WOJDOWSKI}

It is also worth adding that this theorem is true not only for real functions but also for functions with value in an arbitrary topological regular space.

In the sequel, we will consider the topology generated by the so-called lower or almost lower density operator, therefore we briefly recall these notions.

Let $(X, \mathcal{S}, \mathcal{J})$ be a measurable space where $\mathcal{S}$ is a $\sigma$-field and $\mathcal{J} \subset \mathcal{S}$ is a proper $\sigma$-ideal.

We shall say that an operator $\Phi: \mathcal{S} \rightarrow \mathcal{S}$ is a lower density operator on $(X, \mathcal{S}, \mathcal{J})$ if

(1) $\Phi(\emptyset)=\emptyset, \Phi(X)=X$;

(2) $\forall_{A, B \in \mathcal{S}} \Phi(A \cap B)=\Phi(A) \cap \Phi(B)$;

(3) $\forall_{A, B \in \mathcal{S}}(A \triangle B \in \mathcal{J} \Rightarrow \Phi(A)=\Phi(B))$;

(4) $\forall_{A \in \mathcal{S}} \Phi(A) \triangle A \in \mathcal{J}$.

Replacing condition (4) with its weaker form

$\left(4^{\prime}\right) \forall_{A \in \mathcal{S}} \Phi(A) \backslash A \in \mathcal{J}$,

we get the so-called almost lower density operator on $(X, \mathcal{S}, \mathcal{J})$.

We shall say that the pair $(\mathcal{S}, \mathcal{J})$ has the hull property if

$$
\underset{A \subset X}{\forall} \underset{B \in \mathcal{S}, A \subset B}{\exists} \underset{H \subset B \backslash A}{\forall}(H \in \mathcal{S} \Rightarrow H \in \mathcal{J}) .
$$

The following theorem is well-known.

Theorem 2 (cf. [2], 6]). If $\Phi: \mathcal{S} \rightarrow \mathcal{S}$ is a lower density operator on $(X, \mathcal{S}, \mathcal{J})$ and $(\mathcal{S}, \mathcal{J})$ has the hull property, then the family

$$
\mathcal{T}_{\Phi}=\{A \in \mathcal{S}: A \subset \Phi(A)\}
$$

is a topology on $X$ such that

a) $A \in \mathcal{J}$ if and only if $A$ is a $\mathcal{T}_{\Phi}$-nowhere dense and $\mathcal{T}_{\Phi}$-closed set;

b) $\mathbb{K}\left(\mathcal{T}_{\Phi}\right)=\mathcal{J}$;

c) $\mathcal{B} a\left(\mathcal{T}_{\Phi}\right)=\mathcal{B}\left(\mathcal{T}_{\Phi}\right)=\mathcal{S}$;

d) $\left\langle X, \mathcal{T}_{\Phi}\right\rangle$ is a Baire space.

If $\Phi$ is an almost lower density operator, then we have the following theorem.

Theorem 3 (cf. [6]). If $\Phi: \mathcal{S} \rightarrow \mathcal{S}$ is an almost lower density operator on $(X, \mathcal{S}, \mathcal{J})$ and $(\mathcal{S}, \mathcal{J})$ has the hull property, then the family

$$
\mathcal{T}_{\Phi}=\{A \in \mathcal{S}: A \subset \Phi(A)\}
$$

is a topology on $X$ such that

a) if $A \in \mathcal{J}$, then $A$ is a $\mathcal{T}_{\Phi}$-nowhere dense and $\mathcal{T}_{\Phi}$-closed set;

b) $\mathbb{K}\left(\mathcal{T}_{\Phi}\right) \supset \mathcal{J}$;

c) if $\mathbb{K}\left(\mathcal{T}_{\Phi}\right)=\mathcal{J}$, then $\mathcal{B}\left(\mathcal{T}_{\Phi}\right) \subset \mathcal{S}$. 


\section{ON SEMIREGULARIZATION OF ABSTRACT DENSITY TOPOLOGIES}

A topology obtained in Theorem [2 is called an abstract density topology generated by operator $\Phi$ on $(X, \mathcal{S}, \mathcal{J})$.

More interesting properties of topologies generated by lower or almost lower density operators one can find in [6]. Moreover, this paper contains examples of abstract density topologies generated by lower density operators and also by almost lower density operators.

Now, we shall consider a topological Baire space $\langle X, \tau\rangle$ and the measurable space $(X, \mathcal{B} a(\tau), \mathbb{K}(\tau))$. It is well-known that the pair $(\mathcal{B} a(\tau), \mathbb{K}(\tau))$ has the hull property. Thus, every lower density operator $\Phi$ on $(X, \mathcal{B} a(\tau), \mathbb{K}(\tau))$ generates an abstract density topology on $(X, \mathcal{B} a(\tau), \mathbb{K}(\tau))$.

We are going to introduce the lower density operator on $(X, \mathcal{B} a(\tau), \mathbb{K}(\tau))$ which is supported by an operator defined only on the family of $\tau$-open sets.

Let $\Phi: \tau \rightarrow 2^{X}$ be an operator satisfying the following conditions:

i) $\Phi(\emptyset)=\emptyset, \Phi(X)=X$;

ii) $\forall_{A, B \in \tau} \Phi(A \cap B)=\Phi(A) \cap \Phi(B)$;

iii) $\forall_{A \in \tau} A \subset \Phi(A)$.

It is easy to prove the following fact:

Remark 1. $\Phi(V) \subset \bar{V}^{\tau}$ for every $V \in \tau$.

Let $\boldsymbol{\Phi}(X, \tau)$ stand for the family of all operators satisfying the above conditions. This set is called the family of all admissible operators on $\tau$.

It is well-known that in a topological Baire space $\langle X, \tau\rangle$ every set $A \in \mathcal{B} a(\tau)$ has the unique representation:

$$
A=G(A) \triangle B
$$

where $G(A)$ is regular open set in $\langle X, \tau\rangle$ and $B \in \mathbb{K}(\tau)$ (see [1]).

Let $\Phi \in \mathbf{\Phi}(X, \tau)$. Let us define the operator $\Phi_{r}: \mathcal{B} a(\tau) \rightarrow 2^{X}$ in the following way:

$$
\underset{A \in \mathcal{B} a(\tau)}{\forall} \Phi_{r}(A)=\Phi(G(A)) .
$$

In the virtue of properties of regular open sets, it is easy to prove the following theorem.

TheOREM 4. For every $\Phi \in \mathbf{\Phi}(X, \tau)$, the operator $\Phi_{r}$ is a lower density operator on $(X, \mathcal{B} a(\tau), \mathbb{K}(\tau))$ and the family

$$
\mathcal{T}_{\Phi_{r}}=\left\{A \in \mathcal{B} a(\tau): A \subset \Phi_{r}(A)\right\}
$$

is an abstract density topology on $(X, \mathcal{B} a(\tau), \mathbb{K}(\tau))$.

Let us notice the following property concerning an arbitrary lower density operator on $(X, \mathcal{B} a(\tau), \mathbb{K}(\tau))$. 
TheOREM 5. If $\Phi$ is a lower density operator on $(X, \mathcal{B} a(\tau), \mathbb{K}(\tau))$ and the family

$$
\mathcal{T}_{\Phi}=\{A \in \mathcal{B} a(\tau): A \subset \Phi(A)\}
$$

is an abstract density topology on $(X, \mathcal{B} a(\tau), \mathbb{K}(\tau))$, then the following conditions are equivalent:

a) $\tau \subset \mathcal{T}_{\Phi}$;

b) $\forall_{A \in \mathcal{B} a(\tau)} G(A) \subset \Phi(A) \subset \overline{G(A)}^{\tau}$.

Proof. Let $\tau \subset \mathcal{T}_{\Phi}$ and $A \in \mathcal{B} a(\tau)$. Then, $A=G(A) \triangle B$, where $G(A)$ is $\tau$-regular open set and $B \in \mathbb{K}(\tau)$. As $G(A) \in \tau$, we have $G(A) \subset \Phi(G(A))=\Phi(A)$. Simultaneously,

$$
\Phi(A) \backslash \overline{G(A)}^{\tau} \subset \Phi(A) \backslash G(A)=\Phi(G(A)) \backslash G(A) \in \mathbb{K}(\tau) .
$$

It implies that $\Phi(A) \backslash \overline{G(A)}^{\tau} \in \mathcal{T}_{\Phi} \cap \mathbb{K}(\tau)=\{\emptyset\}$. Therefore, $\Phi(A) \subset \overline{G(A)}^{\tau}$, and condition $\mathrm{b})$ has been proved.

Let $V \in \tau$. Then, $V=G(V) \backslash C$, where $C$ is a $\tau$-nowhere dense and $\tau$-closed set (see [11]). Hence, $V \subset G(V) \subset \Phi(G(V))=\Phi(V)$. It implies that $V \in \mathcal{T}_{\Phi}$.

Taking into account Remark 1], we obtain

Corollary 1. If $\Phi \in \mathbf{\Phi}(X, \tau)$, then

$$
\underset{A \in \mathcal{B} a(\tau)}{\forall} G(A) \subset \Phi_{r}(A)=\Phi(G(A)) \subset \overline{G(A)}^{\tau},
$$

and thus, $\tau \subset \mathcal{T}_{\Phi_{r}}$.

Moreover, we have

Remark 2. If $\langle X, \tau\rangle$ is a topological discrete space, then for every operator $\Phi \in \mathbf{\Phi}(X, \tau)$ we get that $\mathcal{T}_{\Phi_{r}}=\tau=2^{X}$.

Remark 3. If $\langle X, \tau\rangle$ is a topological Baire space containing a dense subset $A \in \mathbb{K}(\tau)$ and $\Phi \in \boldsymbol{\Phi}(X, \tau)$, then $X \backslash A \in \mathcal{T}_{\Phi_{r}} \backslash \tau$, so that $\mathcal{T}_{\Phi_{r}} \backslash \tau \neq \emptyset$.

Remark 4. If $\langle X, \tau\rangle$ is a topological Baire space and $\Phi(A)=A$ for every $A \in \tau$, then $\Phi \in \mathbf{\Phi}(X, \tau)$ and $\mathcal{T}_{\Phi_{r}}=\tau \ominus \mathbb{K}(\tau)$.

Proof. Obviously, $\tau \ominus \mathbb{K}(\tau) \subset \mathcal{T}_{\Phi_{r}}$. Let $A \in \mathcal{T}_{\Phi_{r}}$. Then, $A \in \mathcal{B} a(\tau)$ and $A=G(A) \triangle B$, where $B \in \mathbb{K}(\tau)$. Since

$$
A=G(A) \triangle B \subset \Phi_{r}(A)=\Phi(G(A))=G(A),
$$

we get that $A=G(A) \backslash B \in \tau \ominus \mathbb{K}(\tau)$.

In many cases, a topological space $\left\langle X, \mathcal{T}_{\Phi_{r}}\right\rangle$ is not completely regular and even not regular. Namely, we have 


\section{ON SEMIREGULARIZATION OF ABSTRACT DENSITY TOPOLOGIES}

TheOREM 6 (compare [3]). If $\langle X, \tau\rangle$ is a topological Baire space such that there exists a $\tau$-dense set $A \in \mathbb{K}(\tau)$, then for every $\Phi \in \mathbf{\Phi}(X, \tau)$ topological space $\left\langle X, \mathcal{T}_{\Phi_{r}}\right\rangle$ is not regular.

If a topological space $\left\langle X, \mathcal{T}_{\Phi_{r}}\right\rangle$ is $T_{1}$ and not completely regular, then $\mathcal{T}_{\Phi_{r}}$ is not the coarsest topology for which real $\mathcal{T}_{\Phi_{r}}$-continuous functions are continuous. Therefore, we are looking for the coarsest topology $\mathcal{T} \subset \mathcal{T}_{\Phi_{r}}$ such that $\mathcal{C}\left(\left\langle X, \mathcal{T}_{\Phi_{r}}\right\rangle\right)=\mathcal{C}(\langle X, \mathcal{T}\rangle)$

Property 1. Let $\langle X, \tau\rangle$ be a topological Baire space, $\Phi \in \mathbf{\Phi}(X, \tau)$ and let

$$
\begin{aligned}
& \mathcal{T}_{\Phi_{r}^{\prime}}=\{A \subset X: A=V \cup B, V \in \tau, B \subset \Phi(V)\}, \\
& \mathcal{T}_{\Phi_{r}^{\prime \prime}}=\{A \subset X: A=V \cup B, V \in \tau, B \subset \Phi(G(V))\} .
\end{aligned}
$$

Then $\mathcal{T}_{\Phi_{r}^{\prime}}, \mathcal{T}_{\Phi_{r}^{\prime \prime}}$ are topologies on $X$ such that $\mathcal{T}_{\Phi_{r}^{\prime}} \subset \mathcal{T}_{\Phi_{r}^{\prime \prime}} \subset \mathcal{T}_{\Phi_{r}}$.

P r o of. Based on the properties of operator $\Phi$, it is easy to obtain that the families $\mathcal{T}_{\Phi_{r}^{\prime}}, \mathcal{T}_{\Phi_{r}^{\prime \prime}}$ are topologies and $\mathcal{T}_{\Phi_{r}^{\prime}} \subset \mathcal{T}_{\Phi_{r}^{\prime \prime}}$. If $A \in \mathcal{T}_{\Phi_{r}^{\prime \prime}}$, then $A=V \cup B$, where $V \in \tau, B \subset \Phi(G(V))$. Let us assume that $V \cap B=\emptyset$. Then,

$$
B \subset \Phi(G(V)) \backslash V \subset \overline{G(V)} \bar{G}^{\tau} \backslash V=\bar{V}^{\tau} \backslash V .
$$

So, $B \in \mathbb{K}(\tau)$ and $A \in \mathcal{B} a(\tau)$. Simultaneously,

$$
\Phi_{r}(V \cup B)=\Phi_{r}(V)=\Phi(G(V)) \supset G(V) \supset V
$$

and

$$
B \subset \Phi(G(V))=\Phi_{r}(V)=\Phi_{r}(V \cup B) .
$$

Thus, $A=V \cup B \subset \Phi_{r}(V \cup B)=\Phi_{r}(A)$ and $A \in \mathcal{T}_{\Phi_{r}}$.

Remark 5. The inclusions in Property 1 are proper. If $\langle X, \tau\rangle$ is a topological Baire space containing a $\tau$-dense subset $A \in \mathbb{K}(\tau)$, then from Remark 3 for every operator $\Phi \in \boldsymbol{\Phi}(X, \tau)$ we get that $X \backslash A \in \mathcal{T}_{\Phi_{r}} \backslash \mathcal{T}_{\Phi_{r}^{\prime \prime}}$.

If $\Phi(V)=V$ for every $V \in \tau_{0}$, then $\mathcal{T}_{\Phi_{r}^{\prime}}=\tau_{0}$ and $\mathcal{T}_{\Phi_{r}^{\prime \prime}} \backslash \tau_{0} \neq \emptyset$. For example, $A=(-1,1) \backslash \bigcup_{n \in \mathbb{N}}\left\{\frac{1}{n}\right\} \notin \tau_{0}$ but $A=(A \backslash\{0\}) \cup\{0\}$, where $A \backslash\{0\} \in \tau_{0}$ and $\{0\} \in \Phi(G(A \backslash\{0\}))$. Hence, $A \in \mathcal{T}_{\Phi_{r}^{\prime \prime}} \backslash \mathcal{T}_{\Phi_{r}^{\prime}}$.

Property 2. If $\Phi \in \boldsymbol{\Phi}(X, \tau)$, then

$$
\mathcal{T}_{\Phi_{r}^{\prime}} \ominus \mathbb{K}(\tau)=\mathcal{T}_{\Phi_{r}^{\prime \prime}} \ominus \mathbb{K}(\tau)=\mathcal{T}_{\Phi_{r}} .
$$

Pr o of. Obviously, $\mathcal{T}_{\Phi_{r}^{\prime \prime}} \ominus \mathbb{K}(\tau) \subset \mathcal{T}_{\Phi_{r}}$. Let $A \in \mathcal{T}_{\Phi_{r}}$. Then, $A=\Phi_{r}(A) \backslash B$, where $B \in \mathbb{K}(\tau)$ and

$$
\Phi_{r}(A)=\Phi(G(A))=G(A) \cup(\Phi(G(A)) \backslash G(A)) .
$$

Hence, $\Phi_{r}(A) \in \mathcal{T}_{\Phi_{r}^{\prime}}$ and $A \in \mathcal{T}_{\Phi_{r}^{\prime}} \ominus \mathbb{K}(\tau)$.

This property implies the following corollary. 
Corollary 2. If $\Phi \in \mathbf{\Phi}(X, \tau)$, then

$$
\mathcal{C}\left(\left\langle X, \mathcal{T}_{\Phi_{r}^{\prime}}\right\rangle\right)=\mathcal{C}\left(\left\langle X, \mathcal{T}_{\Phi_{r}^{\prime \prime}}\right\rangle\right)=\mathcal{C}\left(\left\langle X, \mathcal{T}_{\Phi_{r}}\right\rangle\right) .
$$

Example 1. In [10], R. O' M a lle y introduced a.e. topology on $\mathbb{R}$. A set $A$ is a.e. open if $A \in \mathcal{T}_{d}$ and $\lambda(A \backslash \operatorname{int}(A))=0$.

Let $\mathcal{T}_{\text {a.e. }}$ be the family of all a.e. open sets. If $\Phi=\Phi_{d}$, then $\Phi \in \boldsymbol{\Phi}\left(\mathbb{R}, \tau_{0}\right)$ and $\mathcal{T}_{\text {a.e. }}=\mathcal{T}_{\Phi_{r}^{\prime}}($ see [15]).

R. O' Malley in [10] proved that the space $\left\langle\mathbb{R}, \mathcal{T}_{\text {a.e. }}\right\rangle$ is completely regular. Thus, in this case, $\mathcal{T}_{\Phi_{r}^{\prime}}$ is the coarsest topology such that

$$
\mathcal{C}\left(\left\langle\mathbb{R}, \mathcal{T}_{\Phi_{r}}\right\rangle\right)=\mathcal{C}\left(\left\langle\mathbb{R}, \mathcal{T}_{\Phi_{r}^{\prime}}\right\rangle\right) .
$$

In the next part, we shall consider the topology $\mathcal{T}_{\Phi_{\text {ro }}}$ included in $\mathcal{T}_{\Phi_{r}^{\prime}}$.

Let $\langle X, \tau\rangle$ be a topological Baire space and $\Phi \in \boldsymbol{\Phi}(X, \tau)$. By $\mathcal{R} \mathcal{O}\left(X, \mathcal{T}_{\Phi_{r}}\right)$ we denote the family of $\mathcal{T}_{\Phi_{r}}$-regular open sets.

Let $\mathcal{T}_{\Phi_{\text {ro }}}$ be a topology with $\mathcal{R O}\left(X, \mathcal{T}_{\Phi_{r}}\right)$ as an open base. Clearly, $\mathcal{T}_{\Phi_{\text {ro }}} \subset \mathcal{T}_{\Phi_{r}}$. The topological space $\left\langle X, \mathcal{T}_{\Phi_{r o}}\right\rangle$ is called the semiregularization of the space $\left\langle X, \mathcal{T}_{\Phi_{r}}\right\rangle$.

There is a useful characterization of the family $\mathcal{R O}\left(X, \mathcal{T}_{\Phi_{r}}\right)$.

Theorem 7 (cf. [6]). A set $A \subset X$ is a $\mathcal{T}_{\Phi_{r}}$-regular open set if and only if $A=\Phi_{r}(A)$.

TheOREM 8. If $\Phi \in \boldsymbol{\Phi}(X, \tau)$, then $\mathcal{T}_{\Phi_{\text {ro }}} \subset \mathcal{T}_{\Phi_{r}^{\prime}}$ and $\mathcal{T}_{\Phi_{\text {ro }}} \ominus \mathbb{K}(\tau)=\mathcal{T}_{\Phi_{r}}$.

P r o o f. Let $A \in \mathcal{R} \mathcal{O}\left(X, \mathcal{T}_{\Phi_{r}}\right)$. By the previous theorem,

$$
A=\Phi_{r}(A)=\Phi(G(A))=G(A) \cup(\Phi(G(A)) \backslash G(A)) .
$$

Since $\Phi(G(A)) \backslash G(A) \subset \Phi(G(A))$, we have $A \in \mathcal{T}_{\Phi_{r}^{\prime}}$.

Obviously, $\mathcal{T}_{\Phi_{r o}} \ominus \mathbb{K}(\tau) \subset \mathcal{T}_{\Phi_{r}}$. If $A \in \mathcal{T}_{\Phi_{r}}$, then $A=\Phi_{r}(A) \backslash B$, where $B \in \mathbb{K}(\tau)$. As $\Phi_{r}(A) \in \mathcal{R O}\left(X, \mathcal{T}_{\Phi_{r}}\right)$, we have $A \in \mathcal{T}_{\Phi_{r o}} \ominus \mathbb{K}(\tau)$.

Corollary 3. $\mathcal{C}\left(\left\langle X, \mathcal{T}_{\Phi_{r o}}\right\rangle\right)=\mathcal{C}\left(\left\langle X, \mathcal{T}_{\Phi_{r}^{\prime}}\right\rangle\right)=\mathcal{C}\left(\left\langle X, \mathcal{T}_{\Phi_{r}^{\prime \prime}}\right\rangle\right)=\mathcal{C}\left(\left\langle X, \mathcal{T}_{\Phi_{r}}\right\rangle\right)$.

In the case of operator $\Phi=\Phi_{d}$ and O'Malley topology $\mathcal{T}_{a . e .}$, we obtain $\mathcal{T}_{\Phi_{\text {ro }}}=\mathcal{T}_{\text {a.e. }}$. Then $\mathcal{T}_{\Phi_{\text {ro }}}$ is the coarsest topology such that $\mathcal{C}\left(\left\langle\mathbb{R}, \mathcal{T}_{\Phi_{\text {ro }}}\right\rangle\right)=$ $\mathcal{C}\left(\left\langle\mathbb{R}, \mathcal{T}_{\text {a.e. }}\right\rangle\right)$.

The following example shows that semiregularization $\mathcal{T}_{\Phi_{\text {ro }}}$ does not have to be the coarsest topology such that $\mathcal{C}\left(\left\langle X, \mathcal{T}_{\Phi_{r o}}\right\rangle\right)=\mathcal{C}\left(\left\langle X, \mathcal{T}_{\Phi_{r}}\right\rangle\right)$.

Example 2. The family $\tau=\{\emptyset, \mathbb{R}, A, B, A \cup B\}$, where $A=(1, \infty)$ and $B=(-\infty,-1)$, is a topology such that $\mathcal{C}(\langle X, \tau\rangle)$ consists of constant functions.

Let $\tau^{\prime}=\tau \ominus J_{\omega}$, where $J_{\omega}$ is the $\sigma$-ideal of countable sets on $\mathbb{R}$. Then, $\tau^{\prime}$ is a topological $T_{1}$ Baire space. 


\section{ON SEMIREGULARIZATION OF ABSTRACT DENSITY TOPOLOGIES}

Putting $\Phi(W)=W$ for $W \in \tau^{\prime}$, we get that $\Phi \in \mathbf{\Phi}\left(\mathbb{R}, \tau^{\prime}\right)$ and, by Remark 4 , $\mathcal{T}_{\Phi_{r}}=\tau^{\prime} \ominus \mathbb{K}\left(\tau^{\prime}\right)$. Hence, by Theorem 1 .

$$
\mathcal{C}\left(\left\langle X, \mathcal{T}_{\Phi_{r}}\right\rangle\right)=\mathcal{C}\left(\left\langle X, \tau^{\prime}\right\rangle\right)=\mathcal{C}(\langle X, \tau\rangle)
$$

Simultaneously, $A \in \mathcal{T}_{\Phi_{r o}}$, because $A$ is $\tau^{\prime}$-regular open and

$$
\Phi_{r}(A)=\Phi(G(A))=G(A)=A .
$$

Similarly, $B \in \mathcal{T}_{\Phi_{r o}}$. So, $\mathcal{T}_{\Phi_{r o}}$ is not the coarsest topology such that

$$
\mathcal{C}\left(\left\langle X, \mathcal{T}_{\Phi_{r o}}\right\rangle\right)=\mathcal{C}\left(\left\langle X, \mathcal{T}_{\Phi_{r}}\right\rangle\right),
$$

because the coarsest topology is $\mathcal{T}=\{\emptyset, \mathbb{R}\}$.

The next theorem shows that under additional assumptions semiregularization $\mathcal{T}_{\Phi_{r o}}$ is the coarsest topology such that

$$
\mathcal{C}\left(\left\langle\mathbb{R}, \mathcal{T}_{\Phi_{r o}}\right\rangle\right)=\mathcal{C}\left(\left\langle\mathbb{R}, \mathcal{T}_{\Phi_{r}}\right\rangle\right) .
$$

To formulate this theorem, we need the concept of an interval set, so we recall this notion.

Either of the sets

$$
\bigcup_{n \in \mathbb{N}}\left(a_{n}, b_{n}\right), \quad \bigcup_{n \in \mathbb{N}}\left[a_{n}, b_{n}\right]
$$

is a right interval set at a point $x_{0}$ if $x_{0}<b_{n+1}<a_{n}<b_{n}$ for $n \in \mathbb{N}$ and $\lim _{n \rightarrow \infty} a_{n}=x_{0}$.

A left interval set at a point $x_{0}$ is defined in the same way.

The union of a right interval set and a left interval set at the same point $x_{0}$ will be called a both interval set at a point $x_{0}$. A set $A$ is an interval set at a point $x_{0}$ if it is a right interval or a left interval or a both interval set at a point $x_{0}$.

TheOREm 9. Let $\Phi \in \boldsymbol{\Phi}\left(\mathbb{R}, \tau_{0}\right)$ and for every set $A \in \tau_{o}$ every $x \in \Phi(A)$ be a both-side accumulation point of $A$. If for every $\tau_{0}$-regular open set $V$ and every $x \in \Phi(V)$ there exists an interval closed set $F$ at $x$ such that $F \subset V$ and $x \in \Phi(\operatorname{int}(F))$, then semiregularization $\mathcal{T}_{\Phi_{\text {ro }}}$ is the coarsest topology such that $\mathcal{C}\left(\left\langle\mathbb{R}, \mathcal{T}_{\Phi_{\text {ro }}}\right\rangle\right)=\mathcal{C}\left(\left\langle\mathbb{R}, \mathcal{T}_{\Phi_{r}}\right\rangle\right)$

P r o o f. (Compare Theorem 2.7.5 in [1]). By Theorem[1]and Theorem 8 , we have $\mathcal{C}\left(\left\langle\mathbb{R}, \mathcal{T}_{\Phi_{\text {ro }}}\right\rangle\right)=\mathcal{C}\left(\left\langle\mathbb{R}, \mathcal{T}_{\Phi_{r}}\right\rangle\right)$. It is sufficient to prove that the topological space $\left\langle\mathbb{R}, \mathcal{T}_{\Phi_{\text {ro }}}\right\rangle$ is completely regular, because then the topology $\mathcal{T}_{\Phi_{\text {ro }}}$ is the coarsest topology for which real $\mathcal{T}_{\Phi_{r o}}$-continuous functions are continuous. Let $A \in \mathcal{T}_{\Phi_{\text {ro }}}$ and $x_{0} \in A$.

We shall define a function $f \in \mathcal{C}\left(\left\langle\mathbb{R}, \mathcal{T}_{\Phi_{\text {ro }}}\right\rangle\right)$ such that

$$
f\left(x_{0}\right)=1 \quad \text { and } \quad(\mathbb{R} \backslash A) \subset f^{-1}(\{0\}) .
$$




\section{JACEK HEJDUK — RENATA WIERTELAK — WOJCIECH WOJDOWSKI}

Clearly, there exists a $\mathcal{T}_{\Phi_{r}}$-regular open set $V$ such that $x_{0} \in V=\Phi_{r}(V) \subset A$. It implies $x_{0} \in \Phi(G(V))$. By assumption, there exists a closed interval set $F$ at $x_{0}$ such that $F \subset G(V)$ and $x_{0} \in \Phi(\operatorname{int}(F))$. Also by the assumption, every point of the set $\Phi(A)$, where $A \in \tau_{0}$, is the both-side accumulation point of $A$. We get that $\Phi_{r}((a, b))=(a, b)$ for every $a<b, a, b \in \mathbb{R}$. Hence, $\tau_{0} \subset \mathcal{T}_{\Phi_{r o}}$. Moreover, $\operatorname{int}(F) \cup\left\{x_{0}\right\} \in \mathcal{T}_{\Phi_{\text {ro }}}$ because

$$
\Phi_{r}\left(\operatorname{int}(F) \cup\left\{x_{0}\right\}\right)=\Phi\left(G\left(\operatorname{int}(F) \cup\left\{x_{0}\right\}\right)\right)=\Phi(\operatorname{int}(F))=\operatorname{int}(F) \cup\left\{x_{0}\right\} .
$$

Define

$$
f(x)=\left\{\begin{array}{ccc}
1 & \text { for } \quad x=x_{0}, \\
\frac{\operatorname{dist}(x, \mathbb{R} \backslash G(V))}{\operatorname{dist}(x, F)+\operatorname{dist}(x, \mathbb{R} \backslash G(V))} & \text { for } \quad x \neq x_{0} .
\end{array}\right.
$$

Since $G(V) \subset \Phi(G(V))=\Phi_{r}(V) \subset A$, we have $(\mathbb{R} \backslash A) \subset \mathbb{R} \backslash G(V) \subset f^{-1}(\{0\})$. Obviously, $f$ is $\tau_{0}$-continuous on $\mathbb{R} \backslash\left\{x_{0}\right\}$ and $f$ is $\mathcal{T}_{\Phi_{\text {ro }}}$-continuous at $x_{0}$, because $\left(\operatorname{int}(F) \cup\left\{x_{0}\right\}\right) \subset f^{-1}(\{1\})$ and $\operatorname{int}(F) \cup\left\{x_{0}\right\} \in \mathcal{T}_{\Phi_{r o}}$.

The above theorem has application for operators:

- $\Phi_{d} \in \boldsymbol{\Phi}\left(\mathbb{R}, \tau_{o}\right)(\mathrm{W}$. W oj d o w s k i, see [15]);

- $\Phi_{I} \in \boldsymbol{\Phi}\left(\mathbb{R}, \tau_{o}\right)$ (E. Łazarow, W. P or ed a, E. W a g ne r- B oj a kows k a, see [8], [12]);

- $\Phi_{\psi} \in \boldsymbol{\Phi}\left(\mathbb{R}, \tau_{o}\right)$ (W. Wilczyńs ki, W. Wojdowski, see [14]);

- $\Phi_{I(J)} \in \boldsymbol{\Phi}\left(\mathbb{R}, \tau_{o}\right)$ ( R. W i e r te la k, see [13]).

We give another example in which Theorem 9 can be applied.

Let $A \in \mathcal{L}$ and $J=\left\{J_{n}\right\}_{n \in \mathbb{N}}$ be a sequence of intervals tending to zero. It means that

$$
\left|J_{n}\right| \underset{n \rightarrow \infty}{\longrightarrow} 0 \wedge c\left(J_{n}\right) \underset{n \rightarrow \infty}{\longrightarrow} 0,
$$

where $c\left(J_{n}\right)$ is a centre of the interval $J_{n}$, for $n \in \mathbb{N}$.

We shall define an operator $\Phi_{J}: \mathcal{L} \rightarrow \mathcal{L}$ in the following way:

$$
x_{0} \in \Phi_{J}(A) \text { if and only if } \lim _{n \rightarrow \infty} \frac{\lambda\left(A \cap\left(J_{n}+x_{0}\right)\right)}{\left|J_{n}\right|}=1 .
$$

If $J=\left\{\left[-\frac{1}{n}, \frac{1}{n}\right]\right\}_{n \in \mathbb{N}}$, then we get an ordinary density point. It was proved that operator $\Phi_{J}$ is an almost lower density operator and the family

$$
\mathcal{T}_{J}=\left\{A \in \mathcal{L}: A \subset \Phi_{J}(A)\right\}
$$

is a topology on $\mathbb{R}$ containing $\tau_{0}$ (see [7]). 


\section{ON SEMIREGULARIZATION OF ABSTRACT DENSITY TOPOLOGIES}

We say that a sequence of intervals

$$
J=\left\{\left[a_{n}, b_{n}\right]\right\}_{n \in \mathbb{N}},
$$

is right-side (left-side) tending to zero if there exists $n_{0} \in \mathbb{N}$ such that $b_{n}>0\left(a_{n}<0\right)$ for $n \geq n_{0}$ and

$$
\lim _{n \rightarrow \infty} \frac{\min \left\{0, a_{n}\right\}}{b_{n}}=0 \quad\left(\lim _{n \rightarrow \infty} \frac{\max \left\{0, b_{n}\right\}}{a_{n}}=0\right) .
$$

A sequence of intervals $J$ is one-side tending to zero if it is right-side or left-side tending to zero.

We have the useful property.

Property 3. Let $J=\left\{\left[a_{n}, b_{n}\right]\right\}_{n \in \mathbb{N}}$ be a sequence of intervals tending to zero. Then, for an arbitrary set $A \in \mathcal{L}$, every point $x \in \Phi_{J}(A)$ is a both-side accumulation point of $A$ if and only if the sequence $J$ is not one-side tending to zero.

P r o of. Necessity. We assume that for an arbitrary set $A \in \mathcal{L}$ every point $x \in \Phi_{J}(A)$ is a both-side accumulation point of $A$. Suppose that sequence $J$ is right-side tending to zero and let $b>0$. Then $0 \in \Phi_{J}([0, b))$, but 0 is not a both-side accumulation point of $[0, b)$. For the sequence $J$ left-side tending to zero, we obtain contradiction in the similar way.

Sufficiency. Let us assume that $J$ is not one-side tending to zero. Let $A \in \mathcal{L}$ and $x \in \Phi_{J}(A)$. Suppose that $0 \in \Phi_{J}(A)$. We show that 0 is left-side accumulation point of $A$. Since $J$ is not right-side tending to zero, we have two cases.

$1^{o}$ There exists a subsequence $\left\{n_{m}\right\}_{m \in \mathbb{N}}$ such that $b_{n_{m}} \leq 0$ for $m \in \mathbb{N}$. Since $0 \in \Phi_{J}(A)$, we have

$$
\lim _{m \rightarrow \infty} \frac{\lambda\left(A \cap\left[a_{n_{m}}, b_{n_{m}}\right]\right)}{\left|\left[a_{n_{m}}, b_{n_{m}}\right]\right|}=1 .
$$

It implies that 0 is a left-side accumulation point of $A$.

$2^{o}$ There exists a subsequence $\left\{n_{m}\right\}_{m \in \mathbb{N}}$ such that $a_{n_{m}}<0<b_{n_{m}}$ and

$$
\lim _{m \rightarrow \infty} \frac{-a_{n_{m}}}{b_{n_{m}}}=\alpha>0 .
$$

Since $0 \in \Phi_{J}(A)$, we have

$$
\lim _{m \rightarrow \infty} \frac{\lambda\left(A \cap\left[a_{n_{m}}, b_{n_{m}}\right]\right)}{\left|\left[a_{n_{m}}, b_{n_{m}}\right]\right|}=1 .
$$

Therefore, 0 is a left-side accumulation point of $A$.

By the similar argument, we prove that if a sequence $J$ is not left-side tending to zero, then 0 is a right-side accumulation point of $A$. 


\section{JACEK HEJDUK — RENATA WIERTELAK — WOJCIECH WOJDOWSKI}

Theorem 10. Let $J=\left\{J_{n}\right\}_{n \in \mathbb{N}}$ be a sequence of closed intervals tending to zero. For every $\tau_{0}$-regular open set $A$ and every $x \in \Phi_{J}(A)$, there exists an open interval set $B \subset A$ at $x$ such that $x \in \Phi_{J}(B)$.

Pro of. If $x \in A$, then the set $B$ exists by Theorem 14 from [5].

Let $A$ be a $\tau_{0}$-regular open set. Then $A=\bigcup_{i \in \mathbb{N}} A_{i}$, where $A_{i}$ are pairwise disjoint open intervals. It is sufficient to consider $0 \in \Phi_{J}(A) \backslash A$. For $n \in \mathbb{N}$ define the set

$$
I(n)=\left\{i \in \mathbb{N}: A_{i} \cap J_{n} \neq \emptyset\right\} .
$$

We have two cases.

$1^{\circ}$ Only a finite number of sets $I(n)$ is infinite. Without loss of generality we can assume that all sets $I(n)$ are finite. Then, we put $B=\bigcup_{n \in \mathbb{N}} \bigcup_{i \in I(n)} A_{i}$. This set is an open interval set, and obviously, $0 \in \Phi_{J}(B)$.

$2^{o}$ There exists a subsequence $\left\{n_{m}\right\}_{m \in \mathbb{N}}$ such that the set $I\left(n_{m}\right)$ is infinite for $m \in \mathbb{N}$ and $I(j)$ is finite for $j \notin \bigcup_{m \in \mathbb{N}}\left\{n_{m}\right\}$. Then, for every $m \in \mathbb{N}$, we can find a finite set $K\left(n_{m}\right) \subset I\left(n_{m}\right)$ such that

$$
\lambda\left(\bigcup_{i \in I\left(n_{m}\right)} A_{i} \cap J_{n_{m}}\right)-\lambda\left(\bigcup_{i \in K\left(n_{m}\right)} A_{i} \cap J_{n_{m}}\right)<2^{-n_{m}-1}\left|J_{n_{m}}\right| .
$$

For $j \notin \bigcup_{m \in \mathbb{N}}\left\{n_{m}\right\}$, we put $K(j)=I(j)$. Then, we define $B=\bigcup_{n \in \mathbb{N}} \bigcup_{i \in K(n)} A_{i}$. As in the case $1^{o}$, this set is an open interval set. Moreover, $0 \in \Phi_{J}(B)$. Indeed,

$$
\begin{aligned}
\lambda\left(B \cap J_{n}\right) & \geq \lambda\left(\bigcup_{i \in K(n)} A_{i} \cap J_{n}\right)>\lambda\left(\bigcup_{i \in I(n)} A_{i} \cap J_{n}\right)-2^{-n-1}\left|J_{n}\right| \\
& =\lambda\left(A \cap J_{n}\right)-2^{-n-1}\left|J_{n}\right| .
\end{aligned}
$$

Hence,

$$
\lim _{n \rightarrow \infty} \frac{\lambda\left(B \cap J_{n}\right)}{\left|J_{n}\right|}>\lim _{n \rightarrow \infty} \frac{\lambda\left(A \cap J_{n}\right)-2^{-n-1}\left|J_{n}\right|}{\left|J_{n}\right|} .
$$

Since $0 \in \Phi_{J}(A)$, it follows that $0 \in \Phi_{J}(B)$.

Paper [5] contains the following theorem helpful in our considerations.

TheOREM 11. Let $J=\left\{J_{n}\right\}_{n \in \mathbb{N}}$ be a sequence of closed intervals tending to zero and $A$ be an open interval set at 0 such that $0 \in \Phi_{J}(A)$. Then there exists an interval set $B \subset A$ composed of closed intervals such that $0 \in \Phi_{J}(B)$. 


\section{ON SEMIREGULARIZATION OF ABSTRACT DENSITY TOPOLOGIES}

Summarizing Theorems 9, 10, 11 and Property 3, we obtain the following theorem.

Theorem 12. Let $J=\left\{J_{n}\right\}_{n \in \mathbb{N}}$ be a sequence of closed intervals which is not one-side tending to zero. If $\Phi=\Phi_{J}$, then

$$
\Phi \in \mathbf{\Phi}\left(\mathbb{R}, \tau_{o}\right), \quad \tau_{0} \subset \mathcal{T}_{\Phi_{\text {ro }}}
$$

and semiregularization $\mathcal{T}_{\Phi_{r o}}$ is the coarsest topology such that

$$
\mathcal{C}\left(\left\langle\mathbb{R}, \mathcal{T}_{\Phi_{r o}}\right\rangle\right)=\mathcal{C}\left(\left\langle\mathbb{R}, \mathcal{T}_{\Phi_{r}}\right\rangle\right) .
$$

\section{REFERENCES}

[1] CIESIELSKI, K.-LARSON, L.-OSTASZEWSKI, K.: I-density continuous functions, Mem. Amer. Math. Soc. 515 (1994), 133 p.

[2] HAmletT, T. R.-JANKOVIĆ, D.- ROSE, D. A.: Lower density topologies, in: Proc. of the 7th Summer Conf. in honor of M. E. Rudin, Papers on General Topology and Appl. (S. Andima et al., eds.), Madison, WI, USA, 1991, Ann. N. Y. Acad. Sci., Vol. 704, The New York Academy of Sciences, New York, NY, 1993, pp. 309-321.

[3] HEJDUK, J.: On the regularity of topologies in the family of sets having the Baire property, Filomat 27 (2013), 1291-1295.

[4] HEJDUK, J.-LORANTY, A.-WIERTELAK, R.: J-approximately continuous functions, Tatra Mt. Math. Publ. 62 (2015), 45-55.

[5] HEJDUK, J.-LORANTY, A.-WIERTELAK, R.: On $\mathcal{J}$-continuous functions, Tatra Mt. Math. Publ. (to appear).

[6] HEJDUK, J.-WIERTELAK, R.: On the abstract density topologies generated by lower and almost lower density operators, in: Traditional and Present-Day Topics in Real Analysis, Łodź University Press, 2013, pp. 431-447.

[7] HEJDUK, J.-WIERTELAK, R.: On the generalization of density topologies on the real line, Math. Slovaca 64 (2014), 1267-1276.

[8] EAZAROW, E.: The coarsest topology for $\mathcal{I}$-approximately continuous functions, Comm. Math. Univ. Carolinae 27 (1986), 695-704.

[9] MARTIN, N. F. G.: Generalized condensation points, Duke Math. J. 28 (1961), 507-514.

[10] O'MALLEY, R. J.: Approximately continuous functions which are continuos almost everywhere, Acta. Math. Acad. Scient. Hungarice 33 (1979), 395-402.

[11] OXTOBY, J. C.: Measure and Category. Springer-Verlag, Berlin, 1987.

[12] POREDA, E. WAGNER-BOJAKOWSKA,: The topology of $\mathcal{I}$-approximately continuous functions, Rad. Math. 2 (1986), 263-267.

[13] WIERTELAK, R.: On the deep $\mathbb{I}(J)$-density topology, Georgian Math. J. 20 (2013), $817-832$. 


\title{
JACEK HEJDUK — RENATA WIERTELAK — WOJCIECH WOJDOWSKI
}

[14] WILCZYŃSKI, W.-WOJDOWSKI, W.: A category $\psi$-density topology, Cent. Eur. J. Math. 9 (2011), 1057-1066.

[15] WOJDOWSKI, W.: Density topologies involving measure and category, Demonstratio Math. 22 (1989), 797-812.

Received Deccember 2, 2014

\author{
Jacek Hejduk \\ Renata Wiertelak \\ Faculty of Mathematics \\ and Computer Science \\ Łódź University \\ Banacha 22 \\ PL-90-238 Łódź \\ POLAND
}

E-mail: hejduk@math.uni.lodz.pl wiertelak@math.uni.lodz.pl

Wojciech Wojdowski

Institute of Mathematics

Technical University of Łódź

Wólczańska 215

PL-90-924 Eódź

POLAND

E-mail:wojwoj@gmail.com 